stanz von meist mehr als $50 \%$. Dieser hohe Betrag läßt sich keinesfalls durch die mit den beobachteten Frequenzverschiebungen verknüpften geringen Intensitätsänderungen $\Delta\left[\left(v_{0} \pm v_{i}\right)^{4}\right]$ erklären Da die Intensität einer Linie empfindlich mit der Änderung $\left(\partial \alpha / \partial q_{i}\right)_{0}$ der Polarisierbarkeit mit der Kernschwingung verknüpft ist, muß man daher annehmen, daß kleine Änderungen der Kernschwingungen bzw. des Bindungszustandes $u$. U. eine verhältnismäßig große Änderung der Intensitäten zur Folge haben.

Das auffällige Verhalten des Streuvermögens von Aceton gegenüber Methanol, das bei fast dem gleichen Konzentrationsverhältnis $n_{\mathrm{A}} /\left(n_{\mathrm{A}}+n_{, 1}\right) \sim 0,2$ ein Minimum besitzt, bei dem auch der größte negative Wert des Volumeneffektes liegt (zwischen 0,2 und 0,3), gibt Anlaß zu dem Schluß, daß das Assoziat Aceton-Methylalkohol vorwiegend aus einem Molekül der ersten und vier Molekülen der zweiten Sorte besteht. Man könnte sich das etwa nach Art der Abb. 8 vorstellen. Dann läßt sich auch das Minimum des Streuvermögens von Aceton gegenüber Methylalkohol bei $n_{\mathrm{A}} / n_{\mathrm{V}} \sim 0,2 / 0,8$ wohl dadurch erklären, daß der Faktor $\left(\partial \alpha / \partial q_{i}\right)_{0}$ des Aceton-Moleküls durch dessen „Einbettung“ in Methanolmoleküle im Sinne einer Verkleinerung stärker beeinflußt wird als der entsprechende Faktor der infolge der Assoziation einzeln nicht so stark beeinflußten Moleküle des Methylalkohols.

In Tab. 1 sind die Raman-Schwingungsfrequenzen der. Reinsubstanzen und ihre Intensitäten nach den
Angaben von K. W. F. Kohlrausch ${ }^{5}\left(J_{\mathrm{K}}\right)$ und den hier durchgeführten Messungen $\left(J_{\text {gem }}\right)$ angegeben.

In Tab. 1 fällt auf, daß zunächst für die hohen Frequenzen bei Methylalkohol und Aceton eine wesentlich größere Intensität bestimmt wurde, als sie von Kohlrausch nach früheren Messungen anderer Autoren angegeben wird. Da hier die Schwärzungskurve nur in zwei Wellenlängenbereichen bestimmt

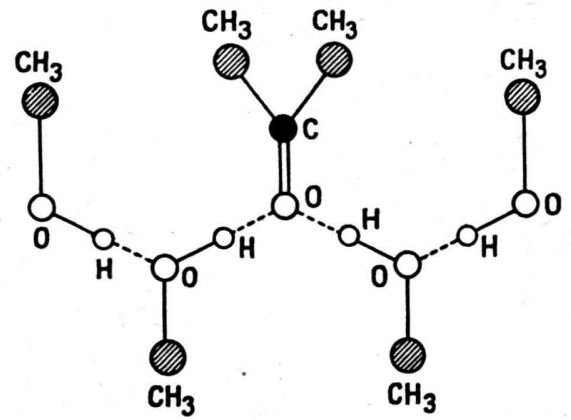

Abb. 8. Assoziation Aceton-Methylalkohol.

wurde, könnte dies auf die nicht genügend berücksichtigte Wellenlängenabhängigkeit der Plattensensibilität zurückzuführen sein. Offenbar aber ist das nicht der einzige Grund, denn es sind in beiden Spektren auch die Intensitätsunterschiede zwischen benachbarten Linien häufig wesentlich größer als die sonst angegebenen Werte, so daß man die Abweichungen zum großen Teil wohl doch auf die früher schon erwähnte unterschiedliche Methode in der Bestimmung der Intensitäten zurückführen muß.

\title{
Die qualitative und quantitative Analyse der isomeren Hexachlorcyclohexane mit Hilfe der Raman-Spektroskopie
}

\author{
Von \\ Horst Luther, Fritz Lampe \\ Institut für chemische Technologie \\ der Technischen Hochschule Braunschweig \\ und \\ Josef Goubeau, Bernhard W. Rodewald \\ Institut für anorganische Chemie \\ der Universität Göttingen
}

(Z. Naturforschg. 5a, 34-40 [1950]; eingegangen am 1. Oktober 1949)

Die Raman-Spektren der isomeren Hexachlorcyclohexane in Tetrahydrofuran und Benzol wurden bestimmt. Aus Gemischen von $\alpha$ - und $\gamma$-Hexachlorcyclohexan verschiedener Zusammensetzung in den genannten Lösungsmitteln wurden Eichkurven für die Veränderung der Intensitätsverhältnisse von Schlüsselfrequenzen der beiden Isomeren mit der Konzentration aufgestellt. Mit Hilfe dieser Eichkurven wurden technische Proben der Isomeren-Gemische analysiert. Die dabei notwendige Vorbehandlung der Substanzen, der Analysengang und die Endauswertung werden beschrieben. Bei einer Dauer einer Analyse zwischen 12 und 20 Stdn. wurde eine Analysengenauigkeit von $\pm 3-4 \%$ absolut erreicht. Die Fehlerquellen werden diskutiert und die Möglichkeiten und Aufgaben einer spektroskopischen Strukturaufklärung der Isomeren behandelt. 


\section{Grundlagen der Methode}

Tn einer vorhergehenden Arbeit ${ }^{1}$ über die alkalische Zersetzung der Hexachlorcyclohexan-Isomeren wurde unter den Verfahren für ihre Analyse bereits die quantitative Raman-Spektralanalyse erwähnt.

Die Grundlage der Methode bildeten die Arbeiten von $\mathrm{G}$ o u b e a u und Mitarbb. ${ }^{2}$, die die quantitative Raman-Analyse auf der Bestimmung der Intensitätsverhältnisse von Schlüsselfrequenzen aufgebauthatten.

Die vorbereitenden Arbeitsgänge sind dabei folgende:

1. Aus den Spektren der später im Gemisch zu analysierenden Reinsubstanzen werden Schlüsselfrequenzen ausgewählt, die sich dadurch auszeichnen, daß sie starke Intensität besitzen und daß sie nicht auch in den Spektren der anderen Gemischteilnehmer auftreten.

2. Da die Additivität der Intensitäten in Gemischen zweier oder mehrerer Substanzen meist nicht gewahrt ist, wẹrden die Intensitäten der Schlüssellinien von Eichmischungen der Analysensubstanzen experimentell bestimmt.

3. Die äußeren Aufnahmebedingungen, wie u. a. Energie des Primärlichtes, Temperatur oder Plattenempfindlichkeit, können nicht bei allen Raman-Aufnahmen absolut konstant gehalten werden. Infolgedessen wäre es schwierig, quantitativ vergleichbare Intensitätsmessungen ohne zusätzliche Arbeitsgänge oder empirische Korrekturen durchzuführen. Die Grundlagen dafür haben erst die Vorschläge von Goubeau und $\mathrm{Otting}{ }^{3}$ sowie von Glockler ${ }^{4}$ über die Doppelröhrchen-Methode und das Ranksche Registrierverfahren ${ }^{5}$ gebracht. Dagegen werden die Intensitätsverhältnisse zweier Schlüssellinien je nach prozentualer Zusammensetzung ihres Gemisches untereinander oder zu der Schlüssellinie eines immer in gleicher Menge vorhandenen Lösungsmittels von den äußeren Bedingungen weitgehend unabhängig sein. Die gemessenen Linienintensitäten werden also als Intensitätsverhältnisse in ihrer Abhängigkeit von der prozentualen Zusammensetzung in Eichkurven dargestellt, aus denen über die Intensitätsverhältnisse

${ }^{1}$ H. Lu ther, H. Ko elbel, E. Rus chen burg u. F. L a m p e, Z. Naturforschg. 4 b, 133 [1949].

${ }^{2} \mathrm{~J}$. G o u b e a u. L. Th a ler, Beih. Angew. Chem. $1941,41$.

3 W. Otting, Diss. Göttingen 1947.

4 G. Glockler u. Yo-Yun Tung, J. chem. Physics 15, 112 [1947].

5 D. H. Rank u. R. V. Wiegand, J. opt. Soc. America 36, 325 [1946]; M. R. F e n s k e u. Mitarb., Analyt. Chem. 19, 700 [1947]. von Schlüsselfrequenzen unbekannter Gemische dann die prozentuale Zusammensetzung abgelesen werden kann.

\section{Die Reinsubstanzen und ihre} Spektren

a) Die Gewinnung. Die Gewinnung und Reinigung der Ausgangssubstanzen wurde bereits in der eingangs erwähnten Arbeit ${ }^{1}$ behandelt. Das von B a s t i a n s e $\mathrm{n}$ und $\mathrm{H}$ a s s e ${ }^{6}{ }^{6}$ beschriebene sogenannte $\zeta$-Isomere mit Schmelzpunkt $145^{\circ}$ wurde aus den Chlorierungsprodukten des Hexachlorcyclohexans isoliert. Über das Ergebnis der Raman- und Ultrarotanalyse dieser Substanz, die kein Isomeres der normalen Hexachlorcyclohexane ist, wird später berichtet werden.

b) Die Aufnahmebedingungen. Da die Hexachlorcyclohexane bei ihren Schmelzpunkten über $100^{\circ} \mathrm{C}$ im Licht der Quecksilberlampe leicht zersetzlich sind, konnten Aufnahmen in der Schmelze nicht gemacht werden. Unter der aus der Kristallisations-Analyse bekannten großen Zahl organischer Lösungsmittel hatten nur das Benzol, das Tetrahydrofuran und das Dimethylformamid $^{7}$ sowohl ein brauchbares Lösungsvermögen für das schwer lösliche $\beta$-Isomere als auch brauchbare optische Eigenschaften und eine geeignete Verteilung der Eigenfrequenzen. In der vorliegenden Arbeit wurden Benzol und Tetrahydrofuran benutzt ${ }^{*}$. Sie wurden unter den mehrfach betriebenen Vorsichtsmaßregeln gereinigt ${ }^{8}$ und extrem getrocknet. Die Reinsubstanzen wurden vor ihrer Lösung gleichfalls noch einmal über Silicagel scharf nachgetrocknet. Eine Trocknung über Phosphorpentoxyd war nicht vorteilhaft, die so getrockneten Substanzen zeigten in Lösung unerwünschte Fluoreszenz. Gelöst wurden je nach den Verhältnissen $10-50 \%$ der Reinisomeren.

Die Aufnahmen wurden in Zeißschen, Steinheilschen und Spezial-Raman-Apparaturen gemacht. Eingestrahlt wurde in Tetrahydrofuran vorwiegend mit der blauen Quecksilberlinie ( $\mathrm{Hg} \mathrm{E}$ bei 4357 $\mathrm{A}$ ), in Benzol vorwiegend mit der violetten Quecksilberlinie (Hg K bei $4047 \AA$ ). Die Belichtungszeiten schwankten bei Verwendung von AgfaSpektralplatten, blau-ultrarapid, je nach verwendeter Apparatur und eingesetztem Filter, in weiten Grenzen zwischen 2 und $20 \mathrm{Stdn}$. Entwickelt wurde mit Standardlösungen unter definierten Bedingungen. Die Schwärzungen wurden auf dem Zeißschen Schnell- und dem Spektrallinien-Photometer gemessen. Die resultierenden Intensitäten wurden über die mit Stufenblendenkondensor bestimmten Schwärzungskurven ermittelt. Von den Gesamtintensitäten der Schlüsselfrequenzen wurden die

${ }^{6}$ O. Bastiansen u. O. Hassel, Acta chem. scand. 1, 683 [1947], Research 2, 248 [1949].

7 N. R. Trenner, R. W. Walker, B. A ris on u. R. P. Buhs, Analyt. Chem. 21, 285 [1949]. 1943.

8 K. W. F. K o h l r a u s h, Ramanspektren, Leipzig

* Die Versuche mit Benzol wurden vorwiegend im Institut für Chemische Technologie der T. H. Braunschweig, die Versuche mit Tetrahydrofuran vorwiegend im Institut für Molekülspektroskopie im Allg. Chem. Institut der Universität Göttingen durchgeführt. 


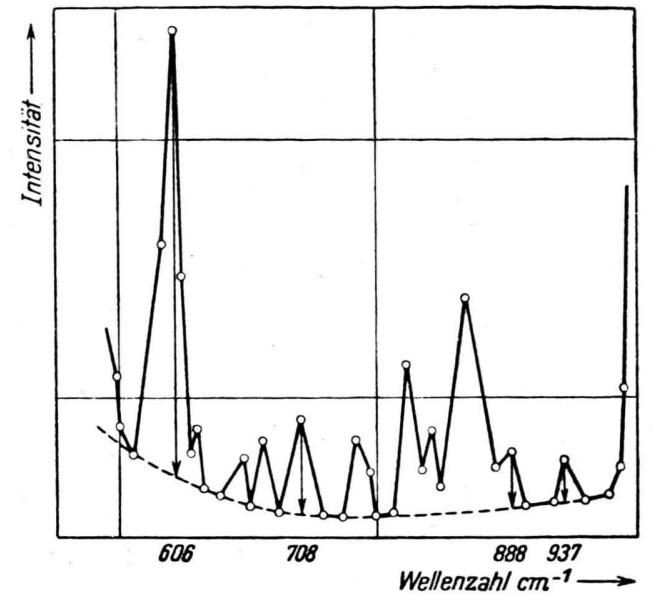

Abb. 1. Linienschwärzungen eines Raman-Spektrums von $2 \mathrm{~g} \gamma$-Hexachlorcyclohexan in $17 \mathrm{~cm}^{3}$ Benzol zwischen 600 und $900 \mathrm{~cm}^{-1}$.

Untergrundintensitäten abgezogen und die Intensitätsverhältnisse der Schlüssellinien gebildet (Abb. 1).

c) Die Spektren der Reinsubstanzen. Im folgenden sind die Spektren des $\alpha-, \beta-, \gamma-, \delta$ - und $\varepsilon$ Isomeren und des als Lösungsmittel benutzten Tetrahydrofurans wiedergegeben. Sie sind Mittel von jeweils mindestens 3 Aufnahmen in Tetrahydrofuran (K- und EFilter) und 3 Aufnahmen in Benzol (K- und E-Filter) (Tab. 1), die Intensitäten wurden in der üblichen Skala zwischen 0 (schwach) und 10 (sehr stark) abgeschätzt. Tab. 2 gibt die für die Aufstellung der Eichkurven verwendeten Schlüsselfrequenzen in Tetrahydrofuran und in Benzol wieder.

\section{Die Spektren der Gemische und die Eichkurven}

Für technische Zwecke steht verständlicherweise der Gehalt eines isomeren Gemisches an der $\gamma$-Komponente im Vordergrund des Interesses. Die quantitative Methode wurde daher nicht für alle Isomeren, sondern vorerst nur für das $\alpha$-, $\gamma$-, und $\delta$-Hexachlorcyclohexan ausgearbeitet. Dabei war die Methodik in Tetrahydrofuran eine andere als in Benzol, um durch vergleichende Messungen methodische Fehler möglichst weitgehend auszuschalten.

a) Die Versuchsbedingungen und die Auswertung in Tetrahydrofuran. Folgende Gemische der reinen $\alpha$ - und $\gamma$-Komponente wurden in getrocknetem Tetrahydrofuran gelöst:

$$
\begin{aligned}
& \alpha: \gamma=10 \quad: \quad 0 \quad \text { Gew.Tle. } \\
& \alpha: \gamma=8 \quad: 2 \quad, \quad, \\
& \alpha: \gamma=6,66: 3,33,, \quad, \\
& \alpha: \gamma=5 \quad: 5 \quad, \quad, \\
& \alpha: \gamma=2: 8 \quad, \quad, \\
& \alpha: \gamma=0 \quad: 10 \quad, \quad,
\end{aligned}
$$

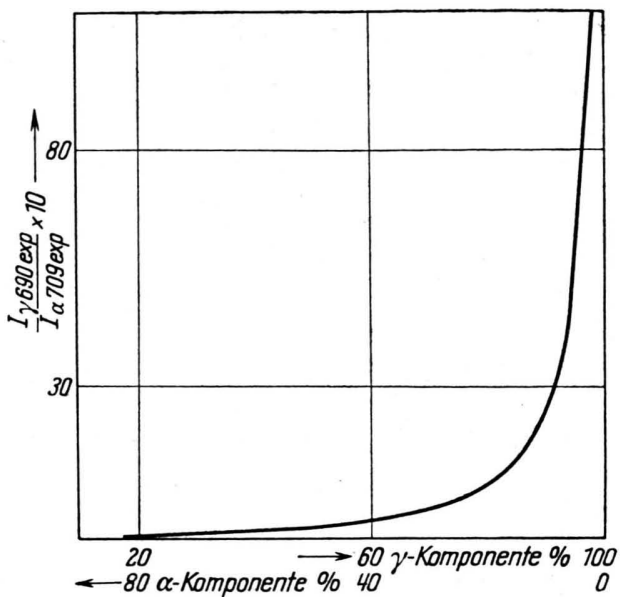

Abb. 2. Eichkurve I zur Bestimmung des $\gamma$-Hexachlorcyclohexans in Tetrahydrofuran als Lösungsmittel.

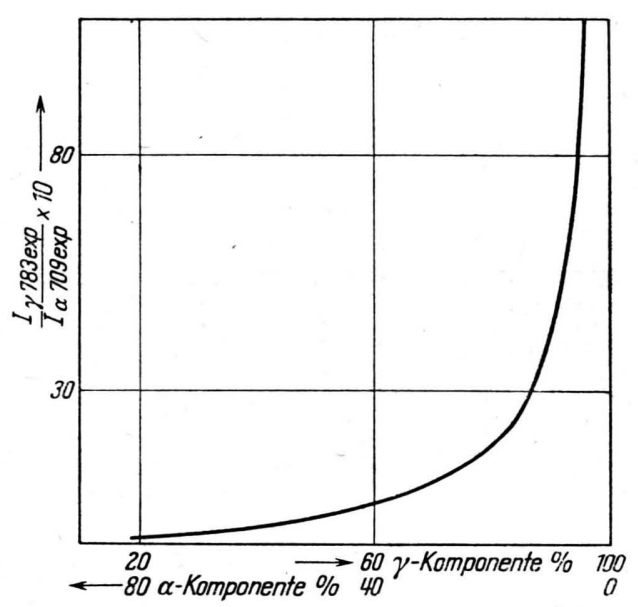

Abb. 3. Eichkurve II zur Bestimmung des $\gamma$-Hexachlorcyclohexans in Tetrahydrofuran als Lösungsmittel.

Die Lösungen wurden unter Anregung durch die blaue Hg-Linie (E) ramanspektroskopiert, die Spektren photometriert, die Intensitäten der Schlüsselfrequenzen $708 \mathrm{~cm}^{-1}$ für $\alpha$ und 689 bzw. $785 \mathrm{~cm}^{-1}$ für $\gamma$ bestimmt und die Intensitätsverhältnisse 708/ 689 und 708/785 gebildet, wobei die Intensitäten stets auf $I_{708}=10$ bezogen wurden. Die sich ergebenden Werte $I_{\gamma}=\frac{I_{\gamma} \exp \times 10}{I_{a} \exp }$ wurden in einem Diagramm als Ordinaten über der Konzentration aufgetragen. Die erhaltenen Eichkurven geben die Abb. 2 und 3 wieder.

b) Die Versuchsbedingungen und die Auswertung in Benzol. Während in Tetrahydrofuran die Intensitäten der Schlüsselfrequenzen der zu analysierenden Substanzen auf einander be- 


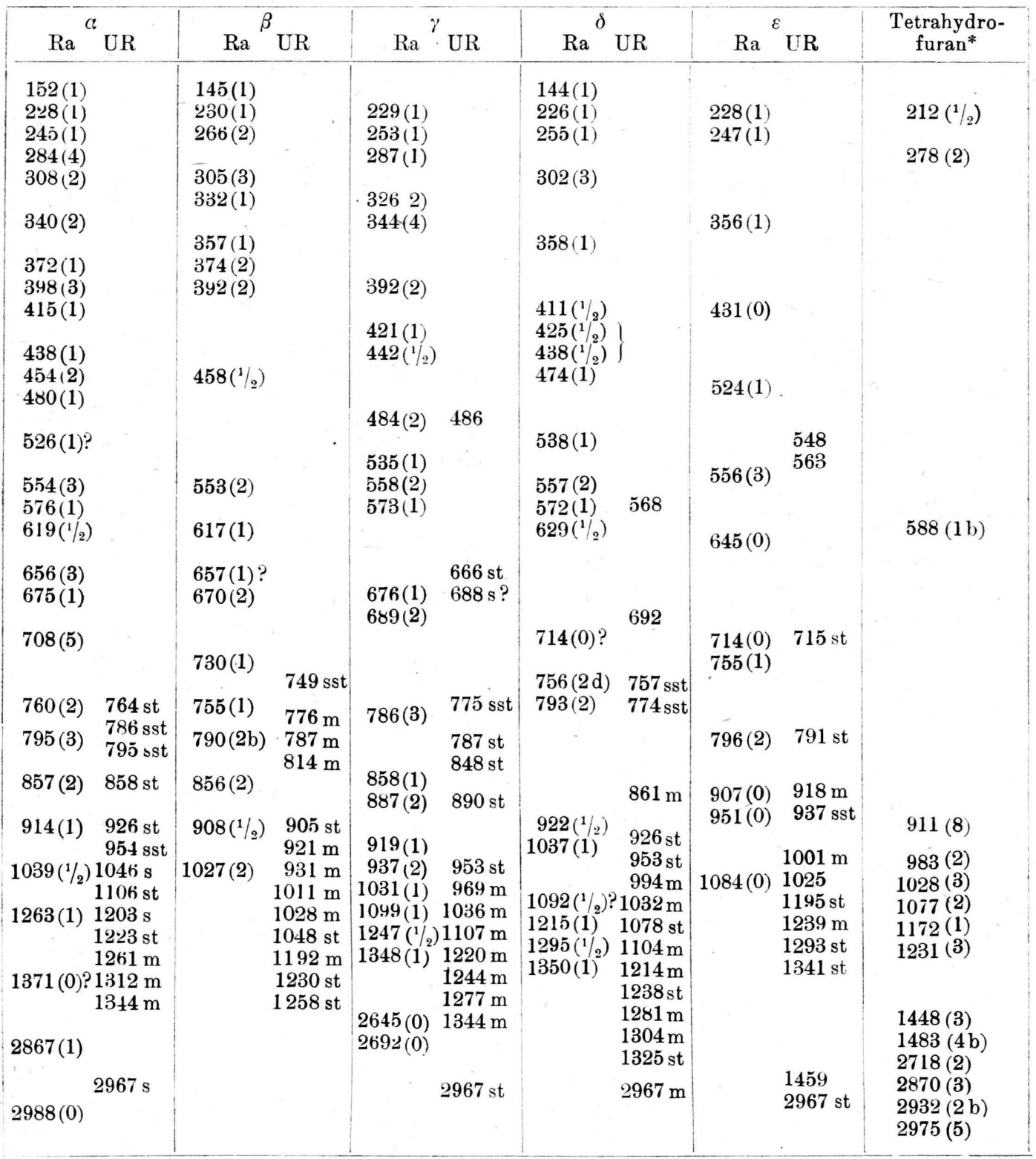

Tab. 1. Die Raman-Spektren der isomeren Hexachlorcyclohexane.

\begin{tabular}{|c|c|c|c|c|c}
\hline $\begin{array}{c}\text { Lösungs- } \\
\text { mittel }\end{array}$ & $\alpha$ & $\beta$ & $\gamma$ & $\delta$ & $\varepsilon$ \\
\hline $\begin{array}{c}\text { Tetrahydro- } \\
\text { furan . . . }\end{array}$ & 708 & $\mathbf{7 3 0}$ & $\mathbf{6 8 9 , 7 8 5}$ & $\mathbf{4 7 4 , 6 2 9}$ & $\mathbf{5 2 4 , 6 4 5}$ \\
Benzol . . . & 708 & $\mathbf{7 3 0}$ & $\mathbf{6 8 9 , 8 8 7 , 9 3 7}$ & $\mathbf{4 7 4 , 6 2 9}$ & $\mathbf{5 2 4 , 6 4 5}$ \\
\hline
\end{tabular}

Tab. 2. Schlüsselfrequenzen für die Analyse der isomeren Hexachlorcyclohexane.

zogen wurden, wurden sie in Benzol auf die Schlüsselfrequenz des Lösungsmittels bei $606 \mathrm{~cm}^{-1}$ bezogen. Dementsprechend wurde jeweils die gleiche Menge
Benzol $\left(17 \mathrm{~cm}^{3}\right)$ vorgelegt und in $\mathrm{ihm} 2 \mathrm{~g}$ des in seiner Zusammensetzung variierenden Gemisches zugesetzt. Für die Bestimmung von $\alpha$ - und $\gamma$-Isomeren wurden die Intensitäten 606 (Benzol), $708(\alpha), 887$ $\left(\gamma_{1}\right), 937\left(\gamma_{2}\right) \mathrm{cm}^{-1}$ bei Anregung durch die violette Hg-Linie $\mathrm{K}$ gemessen. Für die Eichkurven wurden die Intensitätsverhältnisse $I 606 / I 708$ für $\alpha ; I 606 /$ I 887 für $\gamma_{1}$ und $I 606 / I 937$ für $\gamma_{2}$ aufgetragen (Abb. 4-6).

* Vgl. R. L o m ba rd, Bull. Soc. chim. France (5) 14, 523 [1947]. 


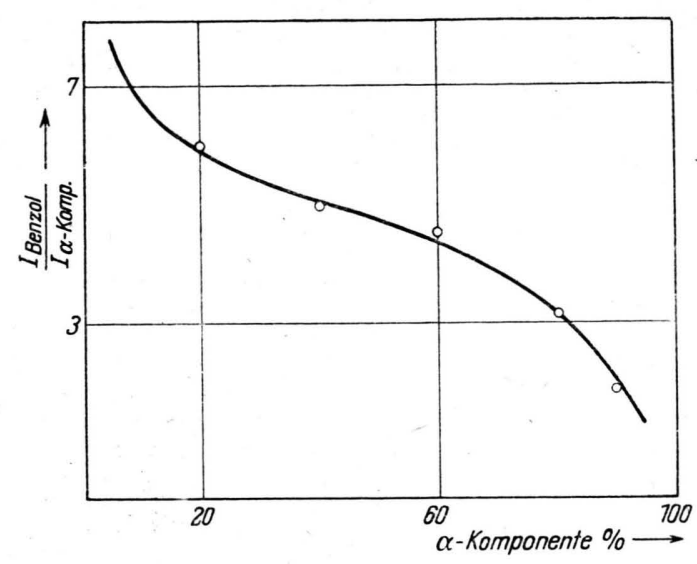

Abb. 4. Eichkurve zur Bestimmung des $\alpha$-Hexachlorcyclohexans in Benzol als Lösungsmittel.

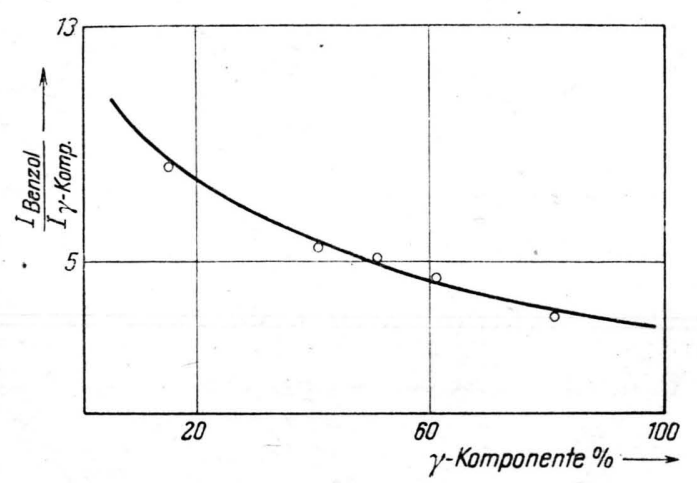

Abb. 5. Eichkurve I zur Bestimmung des $\gamma$-Hexachlorcyclohexans in Benzol als Lösungsmittel.



Abb. 6. Eichkurve II zur Bestimmung des $\gamma$-Hexachlorcyclohexans in Benzol als Lösungsmittel.

\section{Gang einer technischen Analyse}

- Aus der Erfahrung einer großen Reihe durchgeführter technischer Analysen hat sich vorläufig folgender Analysengang ergeben.

\begin{tabular}{|c|c|c|c|c|}
\hline & $a$ & $\beta$ & $\gamma$ & $\delta$ \\
\hline Temperatur ${ }^{\circ} \mathrm{C} .$. & 20 & 20 & 20 & 20 \\
\hline Sulistanzmenge g. & 4 & 1 & 2 & 2 \\
\hline $\mathrm{Al}_{3} \mathrm{O}_{2}-$ Menge g. & 52 & 52 & 26 & 26 \\
\hline Benzollösung $\mathrm{cm}^{3}$. & 100 & 100 & 50 & 50 \\
\hline $\begin{array}{l}\text { Benzolnach- } \\
\text { waichung } \mathrm{cm}^{3} \ldots .\end{array}$ & $2 \times 50$ & $2 \times 50$ & $2 \times 25$ & $2 \times 25$ \\
\hline Verluste g . . . . & 0,28 & 0,1 & 0,90 & 1,1 \\
\hline Verluste $^{0 / 0} \ldots$. & 7 & 10 & 18 & 22 \\
\hline
\end{tabular}

Tab. 3.

Zur Entfernung störender kernchlorierter Produkte wird die Probe mit überhitztem Wasserdampf behandelt. Der Rückstand wird vom Wasser abfiltriert, auf trockenem Filterpapier abgedrückt und unter mäßigem Vakuum über Kieselgel 48 Stdn. getrocknet. Anschließend werden 2 g Substanz in $20 \mathrm{~cm}^{3}$ des gewünschten Lösungsmittels gelöst, nachdem der Gesamtgehalt an Hexachlorcyclohexanen durch die Messung des abspaltbaren Chlors ${ }^{1}$ bestimmt worden ist. Ein evtl. Rückstand kann aus Verunreinigungen oder nicht gelöster $\beta$-Komponente bestehen. $\mathrm{Er}$ wird in einer Parallelprobe bestimmt. Besteht er tatsächlich aus $\beta$-Hexachlorcyclohexan, so kann entsprechend der Löslichkeit von $1,12 \mathrm{~g} \beta$-Komponente in $100 \mathrm{~g}$ Benzol bei $20^{\circ} \mathrm{C}$ und $14,8 \mathrm{~g} \beta$-Isomeren in $100 \mathrm{~g}$ Tetrahydrofuran bei $20^{\circ} \mathrm{C}$ der Gehalt an $\beta$ direkt angegeben werden.

Die meist noch gefärbte Lösung wird - unter Feuchtigkeitsabschluß $\left(\mathrm{CaCl}_{2}\right.$-Röhrchen) - durch eine trockene Schicht Aluminumoxyd nach Brockmann (bei $360^{\circ}$ und $5 \mathrm{~mm} 5$ Stdn. ausgeheizt) von $18 \mathrm{~mm}$ Durchmesser und $10 \mathrm{~mm}$ Höhe (entspr. etwa 2,6 $\mathrm{g} \mathrm{Al}_{2} \mathrm{O}_{3}$ ) filtriert und dabei meist völlig entfärbt. Die auftretenden Substanzverluste insgesamt werden in der vorstehend erwähnten ParallelProbe bestimmt.

Die Verluste an den einzelnen Isomeren können nach den Angaben der Tab. 3 annähernd berücksichtigt werden. Für die in ihr verzeichneten Werte wurden die Reinsubstanzen unter den in Tab. 3 wiedergegebenen Bedingungen durch eine Säule Aluminiumoxyd von $18 \mathrm{~mm}$ Durchmesser und $200 \mathrm{~mm}$ Höhe $\left(52 \mathrm{~g} \mathrm{Al}_{2} \mathrm{O}_{3}\right)$ gesaugt. Anschließend wurde mit Benzol zweimal nachgewaschen. Nach Abdampfen des Lösungsmíttels wurde der Substanzverlust durch Rückwägung festgestellt.

Entsprechend den höheren Konzentrationen an Substanz bei kleinerer Adsorbensmenge können also durch die Reinigung am Aluminiumoxyd etwa 2,5\% der tatsächlich vorhandenen $\gamma$-Komponente verlorengehen. Bis zu $\gamma$ Gehalten von 50\% liegt dieser Fehler auf jeden Fall innerhalb der sonstigen Fehlergrenzen der Methode.

Aus Tetrahydrofuran sind die färbenden Begleitstoffe der Analysensubstanzen nicht so gut an Aluminiumoxyd adsorbierbar wie aus Benzol. Hier muß die Schichthöhe teilweise größer gewählt werden. Aber auch bei größerer Aluminiumoxydmenge ist dieses der Aktivkohle vorzuziehen, da die Adsorbierbarkeit der Hexachlorcyclohexane an Aktivkohle größer ist.

Das Adsorbens wird bei nicht allzu starker Belastung durch verfärbende Begleitstoffe zweimal mit je $2 \mathrm{~cm}^{3}$ Lösungsmittel nachgewaschen. Die Verdampfungsverluste an Lösungsmittel bei diesen Arbeitsgängen betragen 5 bis 
$10 \mathrm{~cm}^{3}$. Die gereinigte Lösung wird in ein Raman-Röhrchen gebracht und auf die an ihm angebrachte. Strichmarke für $17 \mathrm{~cm}^{3}$ aufgefüllt.

Aufnahme des Spektrums, Entwicklung und Photometrierung der Platte werden in bekannter Weise durchgeführt. Abb. 7 gibt die Photometerkurve eines Gemisches wieder.

Es ist zu beachten, daß die Eichkurven in Tetrahydrofuran für Anregung im Blau und in Benzol für Anregung im Violett gelten.

Mit den jeweilig sich ergebenden Intensitätsverhältnissen der Schlüssellinien kann schließlich in die Eichkurven eingegangen werden, um den Gehalt an den betreffenden Isomeren festzustellen.



Abb. 7. Linienschwärzungen des Raman-Spektrums eines normalen technischen Hexachlorcyclohexanproduktes.

Durch den langsamen Anstieg der Eichkurven für Tetrahydrofuran im Gebiet kleiner $\gamma$-Konzentrationen liegt das Optimum ihrer Anwendbarkeit zwischen 25 und $90 \% \gamma$-Komponente. In Benzol dagegen liegt dieses Optimum zwischen 5 und $50 \%$, d. h. also, die beiden Bestimmungsmethoden ergänzen sich gut.

Die Fehlerquellen der gesamten Methode liegen bei technischen Produkten besonders in der Verfärbung und in auftretender Fluoreszenz, die starke Untergrundschwärzungen und damit schlechte und unsichere Photometrierbarkeit verursachen.

In sehr gut vorgetrockneten Proben bewirkt 1\% Nitrobenzol eine gute Fluoreszenzlöschung, beeinflußt aber auch die Streuintensität und die Eigenabsorption der Proben in unübersichtlicher Weise. Bei Anwesenheit geringster Wasserspuren ist seine Wirkung gering oder führt sogar zu sofortigen oder durch das Quecksilberprimärlicht ausgelösten Verfärbungen der Substanz, die besonders im Blau absorbieren.
Für den größten Teil technischer Analysen kann unter Berücksichtigung der verlustbringenden Vorbehandlungsmethoden und der vorstehend diskutierten aufnahmetechnischen Schwierigkeiten mit einer Analysengenauigkeit von $\pm 3-4 \%$ (absolut) gerechnet werden.

In besonders ungünstigen Fällen gelang es, durch Destillation bei $10^{-2}-10^{-3}$ Torr ohne beobachtbare Zersetzungserscheinungen die Proben so weit vorzureinigen, daß brauchbare Aufnahmen entstanden.

Natürlich wurde versucht, die Brauchbarkeit der Methode für technische Proben - für Testgemische steht sie, wie mehrere Analysen ergaben, außer jedem Zweifel -, durch Vergleichsmethoden zu überprüfen. Im Ultrarot treten durch Begleitsubstanzen ähnliche Schwierigkeiten auf, wie sie N. R. Trenner und Mitarbb. $^{7}$ vor kurzem diskutiert haben. Dabei wurden allerdings kernchlorierte Produkte durch die Wasserdampfbehandlung in unserem Falle weitgehend entfernt. Über die Spektren möglicherweise vorhandener Heptáchlorverbindungen, die zu merklichen Fehlern Anlaß geben können, wird später zu berichten sein. Das $\alpha$-Heptachlorcyclohexan besitzt Streulinien in der Nähe der Schlüsselfrequenzen des $\gamma$-Isomeren.

Die Gegentestung des Verfahrens durch Tierversuche ergab in einzelnen Fällen das interessante Resultat, daß durch noch nicht im einzelnen bestimmbare Begleitsubstanzen, die der spektroskopischen Analyse wahrscheinlich durch die Vorbehandlung verlorengehen, die Wirkung des $\gamma$-Isomeren erhöht werden kann, so daß scheinbar mehr $\gamma$-Komponente vorhanden ist, als sich spektroskopisch nachweisen läßt. Wirkungsabschwächungen im Tiertest scheinen gleichfalls vorhanden zu sein, doch ist das dafür vorhandene Material noch nicht eindeutig.

Der Zeitbedarf einer quantitativen Analyse ist wie folgt zu spezifizieren:

1. Vorbehandlung der Substanz

4 Stdn.

2. Belichtungszeit je nach Stärke der Primärlichtquelle

3. Entwickeln, Trocknen, Ausmessen, Photometrieren der Platte

$1-20$ Stdn.

4. Auswertung der Ergebnisse

5 Stdn. 2 Stdn.

Unter 12 Stdn. dürfte also die Zeit für eine Analyse kaum zu drücken sein. Dabei macht es wenig aus, ob eine oder mehrere Komponenten bestimmt werden sollen.

\section{Die Struktur der Isomeren}

Die Ergebnisse modellmäßiger Betrachtungen über die Zahl der möglichen isomeren Hexachlorcyclohexane, die sich sowohl von der Sessel- als auch von 
1

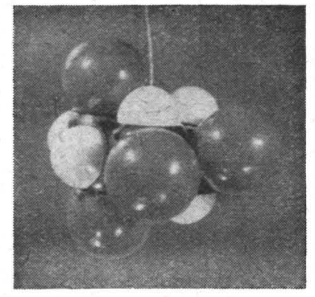

3

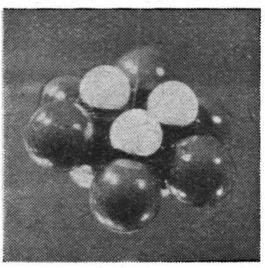

5



7

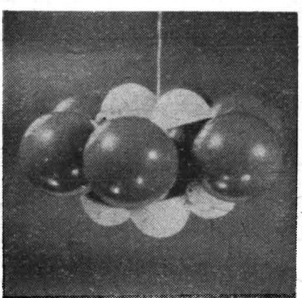

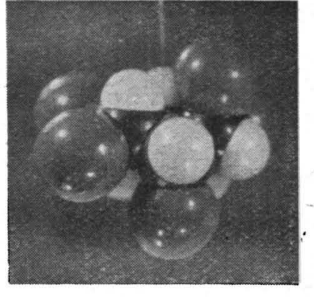
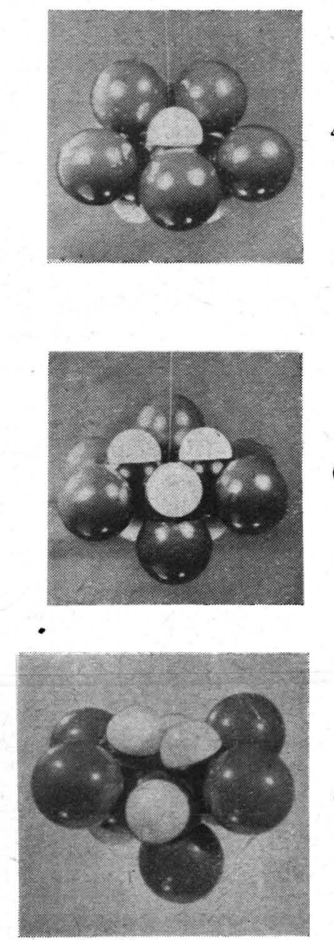
suchungen und die Ergebnisse van V loten $\mathrm{s}^{10}$ änderten B a s ti a n s e n und $\mathrm{H}$ a s s e ${ }^{6}{ }^{6}$ (1949) die bisherigen Zuordnungen ab. Ihre Vorstellungen, die in Abb. 8 mit den Stuart-Modellen verdeutlicht und in Tab. $4^{*}$ zusammengestellt sind, entsprechen auch den Dipolmomenten und Spektren besser als die bisherigen.

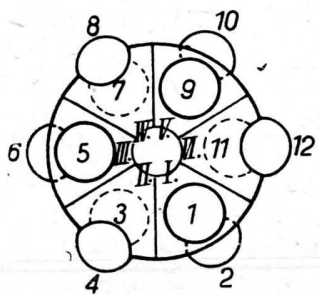

Abb. 9.

Nach den Modellen besitzen das $\beta$ - und $\varepsilon$-Isomere die höchste Symmetrie; sie sollten das Dipolmoment Null und die linienärmsten Spektren besitzen. In

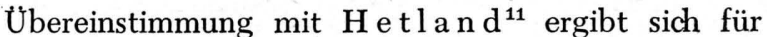
die beiden genannten Isomeren das Dipolmoment Null ${ }^{12}$; wie Tab. 4 zeigt, sind ihre Raman- und Ultrarot-Spektren tatsächlich die linienärmsten.

Eine eingehende kritische Besprechung der Spektren im Hinblick auf strukturelle Fragen soll einer weiteren Arbeit vorbehalten bleiben.

Den HHrn. Dr. H. K o e l b e l und Dr. E. R u s c h e nburg, Forschungslaboratorium Homberg der $\mathrm{Chem}$. Werke Rheinpreußen, dankt der eine von uns ( $\mathrm{L}$ a m p e) für ihr förderndes Interesse.

* Nach B a stia n s e $\mathrm{n}$ und $\mathrm{H}$ a s s e $\mathrm{l}$ (Tidsskr. Kjemi Bergves. 8, 96 [1946]) sind in der Molekülebene liegende Atome mit $\mathrm{k}$, senkrecht $\mathrm{zu}$ ihr liegende mit $\varepsilon$ bezeichnet. Die Zählung der Tab. 4 beginnt, der schematischen Darstellung der Abb. 9 folgend, jeweils bei der in den Bildern vorn stehenden Gruppe. 